\title{
van der Waals corrected DFT simulation of adsorption processes on transition-metal surfaces: $\mathrm{Xe}$ and graphene on $\mathrm{Ni}(111)$
}

\author{
Pier Luigi Silvestrelli and Alberto Ambrosetti \\ Dipartimento di Fisica e Astronomia, Università di Padova, via Marzolo 8, I-35131 Padova, Italy \\ and DEMOCRITOS National Simulation Center, of the Italian Istituto Officina dei Materiali (IOM) \\ of the Italian National Research Council (CNR), Trieste, Italy \\ (Received 14 January 2015; revised manuscript received 20 April 2015; published 6 May 2015)
}

\begin{abstract}
The DFT/vdW-WF2s1 method, recently developed to include the van der Waals interactions in the density functional theory and describe adsorption processes on metal surfaces by taking metal-screening effects into account, is applied to the case of the interaction of Xe and graphene with a transition-metal surface, namely, $\mathrm{Ni}(111)$. In general, the adsorption of rare-gas atoms on metal surfaces is important because it is prototypical for physisorption processes. Moreover, the interaction of graphene with $\mathrm{Ni}(111)$ is of great interest for practical applications, for instance concerning the efficient and large-scale production of high-quality graphene; from a theoretical point of view, it is particularly challenging, since it can be described by a delicate interplay between chemisorption and physisorption processes. The first-principles simulation of transition metals requires particular care also because they can be viewed as intermediate systems between simple metals and insulating crystals. Even in these cases the method performs well as demonstrated by comparing our results with available experimental data and other theoretical investigations. We confirm that the rare-gas Xe atom is preferentially adsorbed on the top-site configuration on the $\mathrm{Ni}(111)$ surface too. Our approach, based on the use of the maximally localized Wannier functions, also allow us to well characterize the bonds between graphene and $\mathrm{Ni}(111)$.
\end{abstract}

DOI: 10.1103/PhysRevB.91.195405

PACS number(s): 68.43.Bc, 71.15.Mb, 73.22.Pr

\section{INTRODUCTION}

The interaction of graphene with transition-metal surfaces is of great importance because the growth of graphene on these substrates is probably at present the most prospective way for the large-scale preparation and production of highquality graphene [1,2]. In particular, the $\mathrm{Ni}(111)$ surface is very interesting, being the closest matched interface with respect to graphene of all transition metals (with a lattice mismatch of about 1\%), and has been therefore intensively investigated [1-31]. In fact, the close lattice match allows graphene to adapt itself to the $\mathrm{Ni}(111)$ lattice and enables the formation of a well-ordered $p(1 \times 1)$ overstructure on the $\mathrm{Ni}(111)$ surface, which is much simpler than the complex Moiré patterns commonly found in other transition-metal surfaces [1-3]. Moreover, given the ferromagnetic properties of $\mathrm{Ni}$, graphene on $\mathrm{Ni}$ surface has also been proposed as a promising spin-filtering device needed in spintronics [32]. Due to the strong interaction between graphene $\pi$ and $\mathrm{Ni}$ $3 d$ electrons, the graphene electronic structure is heavily modified if compared to the electronic structure of freestanding graphene. The most important change is the gap opening resulting from sublattice symmetry breaking.

From a fundamental point of view, the nature of the bonds at the graphene-metal interface has not been completely elucidated yet. Basically, by taking the $\mathrm{C}-\mathrm{C}$ covalent bonds as a reference, the interaction of graphene with a metal substrate can be classified as "weak" [with $\operatorname{Ir}(111), \mathrm{Au}(111)$, $\mathrm{Cu}(111), \mathrm{Pt}(111), \mathrm{Al}(111)$, and $\mathrm{Pd}(111)$ ] or "strong" [with Ni(111), Co(0001), Rh(111), and Ru(0001)] (see Refs. [3,21], and references therein). Clearly this behavior depends on the atomic and electronic structures of the different substrates which can substantially modify the properties of the adsorbed graphene with respect to those of a freestanding graphene layer. In particular, transition metals can be viewed as intermediate systems between simple metals and insulating crystals because their conduction electrons are of both free-electron $(s$ - and $p$-like) character and of localized ( $d$-like) character, so that they are neither totally free nor completely bound. Moreover, the $d$-like electrons of the filled bands are not rigidly bound to the ionic cores and therefore must be somehow included in the description of screening effects.

From a theoretical point of view the interaction of graphene with $\mathrm{Ni}(111)$ represents a particularly challenging system, since both an accurate description of the metallic surface and of the nonlocal correlation effects is needed. Moreover, in the most favored adsorption configurations, van der Waals $(\mathrm{vdW})$ effects are very important although the graphenemetal interaction is relatively strong and the equilibrium distance of about $2.1 \AA$ [5] is much shorter than typical distances observed between fragments bonded by pure vdW interactions, thus suggesting that a delicate interplay between chemisorption and physisorption exists [19,20]. As a result, the computed adsorption energies and equilibrium distances, even by adopting vdW corrected density functional theory (DFT) approaches, exhibit a disappointing wide spread of values [19]. For instance, using the generalized gradient approximation (GGA) functionals one has a very weak binding or no binding at all (see Ref. [19], and references therein), while the local density approximation (LDA) calculations [19] lead to a pronounced minimum with an adsorption energy of about $-200 \mathrm{meV}$ per $\mathrm{C}$ atom, at an equilibrium distance of $2.00 \AA$, a little shorter than the experimental value of $2.11 \pm 0.07 \AA$ [5]. Actually, the deep LDA minimum is a true artifact, being related to the overestimated LDA exchange contribution. In any case the relatively weak bonding confirms that the adsorption should not be viewed as a traditional (covalent) chemisorption characterized by typical adsorption energies of $0.5-2 \mathrm{eV}$, although the short graphene-Ni distances 
clearly indicate a covalent interaction already at the level of semilocal GGA functionals.

The adsorption of graphene on $\mathrm{Ni}(111)$ has been also investigated on the basis of the adiabatic-connection fluctuation-dissipation theorem in the random phase approximation (RPA) $[20,26]$. At the moment this probably represents the most accurate calculation, which can therefore be taken as a reasonable reference database, being particularly suited to describe intricate bonds with mixed covalent and dispersive character [20,26], although one should remember that RPA typically exhibits a tendency to underbind [33-35]. In spite of a significant hybridization found between the graphene $\pi$ orbitals and $\mathrm{Ni} d_{z^{2}}$ states at a binding distance of about $2.2 \AA$, the computed RPA adsorption energy is still in the range of a typical physisorption interaction (about $-70 \mathrm{meV}$ per $\mathrm{C}$ atom). An important contribution to the energy is related to a decrease in the exchange energy resulting from the adsorption-induced lower symmetry in the graphene layer. Interestingly, the RPA calculations do not only predict one single minimum but also a second minimum at a distance of $3.3 \AA$. This second minimum, with an adsorption energy of $-60 \mathrm{meV}$, that is only slightly less bound than the most favored configuration, is found at a distance typical for vdW-adsorbed graphene on transition metals [36].

This complex scenario has been rationalized as follows [20]: at large distances, namely, up to $2.8 \AA$, the electronic band structure of graphene on $\mathrm{Ni}(111)$ is hardly modified with respect to that of the freestanding, the exchange interaction is purely repulsive, and the correlation follows essentially a vdW-like behavior; instead, at distances shorter than $2.8 \AA$, the graphene band structure starts to be modified with a hybridization setting in and, most importantly, with a breakup of the symmetry between the top-site and hollow-site $\mathrm{C}$ atoms. As a delicate balance between exchange and correlation energy the total energy is smooth, with a slight barrier between the physisorption minimum, characterized by a graphene band structure that is hardly modified compared to freestanding graphene, and another "chemisorption" minimum, where the graphene band structure is strongly modified compared to the freestanding layer. The physisorption minimum originates entirely from vdW forces, whereas the chemisorption minimum can be attributed to both orbital hybridization and week $\mathrm{vdW}$ forces. The slight energy barrier between the physical adsorption and chemisorption states implies that the graphene can be decoupled easily from the $\mathrm{Ni}(111)$ surface, which is also evidenced in chemical vapor deposition growth of graphene on the Ni surface [19]. Besides vdW effects at large distances it is therefore also important to describe exchange interaction at a short distance where the correct description of Pauli repulsion and hybridization is crucial [24].

Since graphene has two carbon atoms in the unit cell, while the $\mathrm{Ni}(111)$ surface has only one, six different structural models are possible, namely, top-fcc, top-hcp, bridge-top, $f c c-h c p$, bridge-fcc, and bridge-hcp. In the top-fcc and top-hcp models, one $\mathrm{C}$ atom sits on top of the Ni atom while the other $\mathrm{C}$ atom occupies, respectively, the fcc and hep hollow sites. The bridge-top configuration has the carbon-carbon bond of graphene on top of a surface $\mathrm{Ni}$ atom. In the fcc-hcp model, both $\mathrm{C}$ atoms are in the threefold hollow sites (see Fig. 1).

Recently, the structural properties of graphene/Ni(111) have been experimentally investigated [21] by a combination (a)

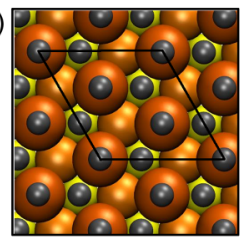

top-fcc

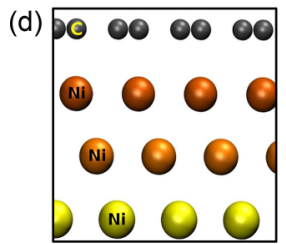

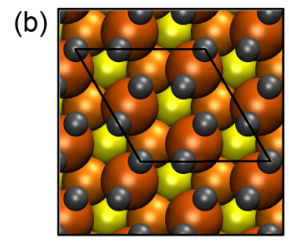

bridge-top

(e)

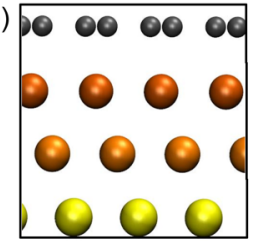

(c)

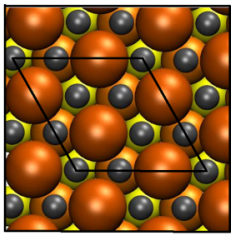

fcc-hcp

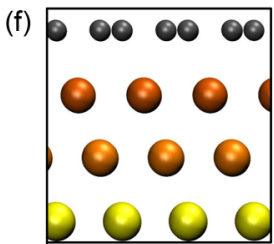

FIG. 1. (Color online) Plane (upper panels) and side (lower panels) views of graphene on the $\mathrm{Ni}(111)$ surface, in the top-fcc, bridge-top, and fcc-hcp configurations; the adopted simulation cell is shown in the plane views.

of low-energy electron diffraction (LEED), x-ray photoelectron spectroscopy (XPS), and angle-scanned photoelectron diffraction. XPS data indicate that graphene interacts strongly with the topmost Ni layer and diffraction data show that graphene is deposited commensurably with the underlying $\mathrm{Ni}$ surface atoms. Interestingly, related first-principles calculations based on DFT show [21] that the total energies of the top-fcc and bridge-top structures are nearly degenerate, which corroborates the observed $[18,21]$ coexistence of those phases. A depletion of charge density in Ni- $d_{z^{2}}$ of the topmost Ni atoms is also found and an increase of that in $p_{z}$ of the on-top $\mathrm{C}$ atom in the top-fcc and top-hcp structures, which indicates that the electrons transfer from substrate to adsorbed graphene [19].

From a theoretical point of view, it is well accepted that the widely used LDA and semilocal GGA functionals fail to capture vdW forces, which are important in rare-gas/metal and graphene/metal systems. As mentioned above, the most reliable RPA calculations $[20,26]$ show that the binding in a graphene/Ni system is a delicate balance between covalent and dispersive interactions. However, the RPA calculation is computationally expensive (scaling as $N^{4}$ with system size) and thus hardly accessible for large systems. The cheaper DFT-D approach [37] with semiempirical corrections for vdW interactions seems to typically give a reasonable prediction of the adsorption properties. However, its empirical character [7,38-40] casts doubts on the full reliability and accuracy in many applications, particularly in a metal system where the atom-based description, implied in the use of the $C_{6}$ coefficients adopted in DFT-D, is rather questionable.

In the last few years several practical methods have been proposed to make DFT calculations able to accurately describe $\mathrm{vdW}$ effects at a reasonable computational cost (for a recent review, see, for instance, Refs. [41-43]). We have developed a family of such methods, all based on the generation of the maximally localized Wannier functions (MLWFs) [44], successfully applied to a variety of systems, including small molecules, water clusters, graphite and graphene, water layers interacting with graphite, interfacial water on semiconducting substrates, hydrogenated carbon nanotubes, molecular solids, and the interaction of rare gases and small molecules with 
metal surfaces [45-57]. Of a particular value is the possibility of dealing with metal surfaces; in fact, insulating surfaces could be somehow treated even using atom-based semiempirical approaches where an approximately derived $R^{-6}$ term, multiplied by a suitable short-range damping function, is explicitly introduced. Instead, in our methods the atom-based point of view assumed in standard semiempirical approaches is replaced by an electron-based point of view, so that the schemes are also applicable to systems, such as metals and semimetals, which cannot be described in terms of assemblies of atoms only weakly perturbed with respect to their isolated configuration.

The DFT/vdW-WF2s1 method, presented in Ref. [55], has been specifically developed to take metal-screening effects into account and has been applied to the study of the adsorption of rare gases and small molecules on different metal surfaces, namely, $\mathrm{Al}(111), \mathrm{Cu}(111)$, and $\mathrm{Pb}(111)$, which are systems where a proper inclusion of screening is essential [58].

Here we apply the DFT/vdW-WF2s1 approach to investigate the interaction of the $\mathrm{Xe}$ atom and of graphene with the $\mathrm{Ni}(111)$ surface. Our results will be compared to the best available, reference experimental and theoretical values, and to those obtained by other DFT vdW corrected schemes, including dispersion corrected Perdew-Burke-Ernzerhof (PBE) (PBE-D [37]), vdW-DF [59,60], vdW-DF2 [61], rVV10 [62], and by the simpler LDA and semilocal GGA (in the PBE flavor [63]) approaches. In the PBE-D scheme DFT calculations at the PBE level are corrected by adding empirical $C_{6} / R^{6}$ potentials with parameters derived from accurate quantum chemistry calculations for atoms, while in other methods, such as vdW-DF, vdW-DF2, and rVV10, vdW effects are included by introducing DFT nonlocal correlation functionals.

\section{METHOD}

Here we briefly review the DFT/vdW-WF2s1 method; additional details can be found in Refs. [54,55]. Basically, the scheme relies on the well-known London expression [64] where two interacting atoms, $A$ and $B$, are approximated by coupled harmonic oscillators and the vdW energy is taken to be the change of the zero-point energy of the coupled oscillations as the atoms approach; if only a single excitation frequency is associated to each atom, $\omega_{A}, \omega_{B}$, then

$$
E_{v d W}^{L \text { ondon }}=-\frac{3 e^{4}}{2 m^{2}} \frac{Z_{A} Z_{B}}{\omega_{A} \omega_{B}\left(\omega_{A}+\omega_{B}\right)} \frac{1}{R_{A B}^{6}},
$$

where $Z_{A, B}$ is the total charge of $A$ and $B$, and $R_{A B}$ is the distance between the two atoms ( $e$ and $m$ are the electronic charge and mass).

Now, adopting a simple classical theory of the atomic polarizability, the polarizability of an electronic shell of charge $e Z_{i}$ and mass $m Z_{i}$, tied to a heavy undeformable ion can be written as

$$
\alpha_{i} \simeq \frac{Z_{i} e^{2}}{m \omega_{i}^{2}} .
$$

Then, given the direct relation between polarizability and atomic volume [65], we assume that $\alpha_{i}=\gamma S_{i}^{3}$, where $\gamma$ is a proportionality constant, so that the atomic volume is expressed in terms of the MLWF spread, $S_{i}$. Rewriting Eq. (1) in terms of the quantities defined above, one obtains an explicit expression for the $C_{6} \mathrm{vdW}$ coefficient:

$$
C_{6}^{A B}=\frac{3}{2} \frac{\sqrt{Z_{A} Z_{B}} S_{A}^{3} S_{B}^{3} \gamma^{3 / 2}}{\left(\sqrt{Z_{B}} S_{A}^{3 / 2}+\sqrt{Z_{A}} S_{B}^{3 / 2}\right)} .
$$

The constant $\gamma$ can then be set up by imposing that the exact value for the $\mathrm{H}$ atom polarizability $\left(\alpha_{\mathrm{H}}=4.5\right.$ a.u. $)$ is obtained; of course, in the $\mathrm{H}$ case, one knows the exact analytical spread, $S_{i}=S_{\mathrm{H}}=\sqrt{3}$ a.u.

In order to achieve better accuracy, one must properly deal with intrafragment MLWF charge overlap. This overlap affects the effective orbital volume, the polarizability, and the excitation frequency [see Eq. (2)], thus leading to a quantitative effect on the value of the $C_{6}$ coefficient. We take into account the effective change in volume due to intrafragment MLWF overlap by introducing a suitable reduction factor $\xi$ obtained by interpolating between the limiting cases of fully overlapping and nonoverlapping MLWFs (see Ref. [54]). We therefore arrive at the following expression for the $C_{6}$ coefficient:

$$
C_{6}^{A B}=\frac{3}{2} \frac{\sqrt{Z_{A} Z_{B}} \xi_{A} S_{A}^{3} \xi_{B} S_{B}^{3} \gamma^{3 / 2}}{\left(\sqrt{Z_{B} \xi_{A}} S_{A}^{3 / 2}+\sqrt{Z_{A} \xi_{B}} S_{B}^{3 / 2}\right)},
$$

where $\xi_{A, B}$ represents the ratio between the effective and the free volume associated with the $A$ th and $B$ th MLWF.

Finally, the vdW interaction energy is computed as

$$
E_{v d W}=-\sum_{i<j} f\left(R_{i j}\right) \frac{C_{6}^{i j}}{R_{i j}^{6}},
$$

where $f\left(R_{i j}\right)$ is a short-range damping function defined as follows:

$$
f\left(R_{i j}\right)=\frac{1}{1+e^{-a\left(R_{i j} / R_{s}-1\right)}} .
$$

We remark that this short-range damping function is introduced not only to avoid the unphysical divergence of the vdW correction at small fragment separations, but also to eliminate double countings of correlation effects, by considering that standard DFT approaches properly describe short-range correlations.

The parameter $R_{S}$ represents the sum of the vdW radii $R_{s}=$ $R_{i}^{v d W}+R_{j}^{v d W}$, with (by adopting the same criterion chosen above for the $\gamma$ parameter)

$$
R_{i}^{v d W}=R_{\mathrm{H}}^{v d W} \frac{S_{i}}{\sqrt{3}},
$$

where $R_{\mathrm{H}}^{v d W}$ is the literature [66] (1.20 $\AA$ ) vdW radius of the $\mathrm{H}$ atom and, following Grimme et al. [67], $a \simeq 20$; note that the results are almost independent of the particular value of this parameter. Although this damping function introduces a certain degree of empiricism in the method, we stress that $a$ is the only ad hoc parameter present in our approach, while all the others are only determined by the basic information given by the MLWFs, namely, from first-principles calculations.

To get an appropriate inclusion of metal-screening effects a proper reduction coefficient is included by multiplying the $C_{6}^{i j} / R_{i j}^{6}$ contribution in Eq. (5) by a Thomas-Fermi factor: $f_{T F}=e^{-2\left(z_{s}-z_{l}\right) / r_{T F}}$ where $r_{T F}$ is the Thomas-Fermi screening length relative to the electronic density of an effective uniform 
electron gas ("jellium model") describing the substrate (see discussion below), $z_{s}$ is the average vertical position of the topmost metal atoms, and $z_{l}$ is the vertical coordinate of the Wannier function center (WFC) belonging to the substrate ( $l=i$ if it is the $i$ th WFC which belongs to the substrate, otherwise $l=j$ ); the above $f_{T F}$ function is only applied if $z_{l}<z_{s}$, otherwise it is assumed that $f_{T F}=1$ (no screening effect).

\section{Computational details}

We here apply the DFT/vdW-WF2s1 method to the case of adsorption of Xe and graphene on the $\mathrm{Ni}(111)$ surface. All calculations have been performed with the QUANTUM-ESPRESSO $a b$ initio package [68] and the MLWFs have been generated as a postprocessing calculation using the WANT package [69]. Similarly to our previous studies $[52,55]$ we modeled the metal surface using a periodically repeated hexagonal supercell, with a $(\sqrt{3} \times \sqrt{3}) R 30^{\circ}$ structure and a surface slab made of $15 \mathrm{Ni}$ atoms distributed over five layers considering the experimental $\mathrm{Ni}(111)$ lattice constants; note that using PBE as the reference exchange-correlation functional, as we do in our study, the optimized Ni lattice constant is very close to the experimental one: $3.524 \AA$ for bulk fcc Ni to be compared with the experimental value of $3.52 \AA$ (see, for instance, Ref. [22]). Repeated slabs were separated along the direction orthogonal to the surface by a vacuum region of about $25 \AA$ to avoid significant spurious interactions due to periodic replicas. The Brillouin zone has been sampled using a $6 \times 6 \times 1 k$-point mesh. Above one of the surface Ni layers we add a Xe atom or a single graphene layer. We remind that the $\mathrm{Ni}(111)$ surface and the graphene lattice constants are, respectively, 2.489 and $2.46 \AA$, which corresponds to a lattice misfit of about $1.2 \%$, so that one can assume that the graphene layer lattice vectors are commensurate with those of the Ni(111) surface. In this model system, with a Xe atom per supercell, the coverage is $1 / 3$, i.e., one adsorbed adparticle for each three metal atoms in the topmost surface layer. The $(\sqrt{3} \times \sqrt{3}) R 30^{\circ}$ structure has been indeed observed [70] at low temperature by LEED for the case of $\mathrm{Xe}$ adsorption on $\mathrm{Cu}(111)$ and $\mathrm{Pd}(111)$; since this is the simplest commensurate structure for rare-gas monolayers on close-packed metal surfaces and the only one for which good experimental data exist, it was adopted in most of the previous $a b$ initio studies [71-76]. After a preliminary relaxation of the outermost layers of the clean metal surfaces, the metal surface atoms were kept frozen and only the vertical coordinate (perpendicular to the surface) of the Xe atom or the graphene layer was optimized, this procedure being justified by the fact that only minor metal surface atom displacements are observed [72,76-78], and relaxation effects are estimated to be small [79]. Also in the case of graphene, which seems to chemisorb on $\mathrm{Ni}(111)$, the buckling between nonequivalent carbon atoms is negligible, indicating that the adsorbed graphene layer is quite smooth [22]. Given the ferromagnetic character of $\mathrm{Ni}$, spin polarization was taken into account.

We have carried out calculations for various separations of the $\mathrm{Xe}$ atom and the graphene layer, with $\mathrm{Xe}$ adsorbed on the top and hollow high-symmetry sites, and considering the top-fcc, bridge-top, and fcc-hcp configurations for graphene on the metal substrates (see above); the hollow site coincides with the center of the triangle formed by the three surface metal atoms contained in the supercell. For better accuracy, as done in previous applications on adsorption processes [46,47,49,50,52,55], we have also included the interactions of the MLWFs of the adsorbate not only with the MLWFs of the underlying surface, within the reference supercell, but also with a sufficient number of periodically repeated surface MLWFs; in any case, given the $R^{-6}$ decay of the $\mathrm{vdW}$ interactions, the convergence with the number of repeated images is rapidly achieved. Electron-ion interactions were described using ultrasoft pseudopotentials by explicitly including ten valence electrons per $\mathrm{Ni}$ atom. We chose the PBE [63] reference DFT functional, which is probably the most popular GGA functional.

The choice of a suitable effective value for the uniform electron density $n$ or, equivalently, the dimensionless " $r_{s}$ " parameter, $r_{s}=(3 / 4 \pi n)^{1 / 3} / a_{0}$ (where $a_{0}$ is the Bohr radius) associated with the metal substrate to get the Thomas-Fermi length $r_{T F}$, defined above to describe screening effects, deserves a specific comment since we are considering transition metals where, differently from simple metals, the electrons cannot be assumed to be totally free. To address this issue we used the list of values for the effective free-electron density parameter $r_{s}$ provided by Perrot and Rasolt [80] for transition metals. These values were obtained by considering the problem of appropriately describing the ground state properties (particularly those of arbitrary defects) of the mobile part of the electron fluid in the transition metals and defining the response of the "free" mobile part of the electron fluid using an effective $r_{s}$ of a uniform electron gas. The recipe is based on the concept of the metallic response to external low-symmetry perturbations and the obtained picture is that, as expected, the $s$ and $p$ electrons carry the main response to an external potential while the $d$ electrons remain largely unpolarized. From the $r_{s}$ parameter $r_{T F}$ can be evaluated using the following formulas [81]:

$$
\begin{gathered}
k_{F}=\left(\frac{9 \pi}{4}\right)^{1 / 3} \frac{1}{r_{s} a_{0}}, \\
k_{T F}^{2}=4 \pi e^{2} g\left(\epsilon_{F}\right)=\frac{4 m e^{2}}{\pi \hbar^{2}} k_{F}, \\
r_{T F}=1 / k_{T F} \simeq \frac{\sqrt{r_{s}}}{1.563} a_{0},
\end{gathered}
$$

where $k_{F}$ and $k_{T F}$ are the Fermi and Thomas-Fermi wave vector, respectively, and $g\left(\epsilon_{F}\right)=m k_{F} / \hbar^{2} \pi^{2}$ is the density of levels at the Fermi energy $\epsilon_{F}$ in the jellium model. Using the $r_{s}=2.14$ value suggested for Ni by Perrot and Rasolt [80], one obtains a Thomas-Fermi screening length $r_{T F}=0.496 \AA$.

\section{RESULTS AND DISCUSSION}

In Table I we report the binding energy and the equilibrium distance for Xe on $\mathrm{Ni}(111)$. The binding energy, $E_{b}$, is defined as

$$
E_{b}=E_{t o t}-\left(E_{s}+E_{\mathrm{Xe}}\right),
$$

where $E_{s, \mathrm{Xe}}$ represent the energies of the isolated fragments [the $\mathrm{Ni}(111)$ substrate and the $\mathrm{Xe}$ atom] and $E_{\text {tot }}$ is the 
TABLE I. Binding energy $E_{b}$ (in $\mathrm{meV}$ ) and (in parentheses) equilibrium distance $R$ (in $\AA$ ) for Xe adsorbed on $\mathrm{Ni}(111)$ in the top and hollow configurations, using different methods. In square brackets the adsorption energy $E_{a}$ (in $\mathrm{meV}$ ), which takes the Xe$\mathrm{Xe}$ interaction into account (see text). Results are compared with available experimental reference data.

\begin{tabular}{lcc}
\hline \hline Method & Top & Hollow \\
\hline LDA & $-401(2.51)$ & $-382(2.17)$ \\
PBE & $-19(3.93)$ & $-18(3.91)$ \\
PBE-D & $-391(2.76)$ & $-354(3.25)$ \\
rVV10 & $-284(3.33)$ & $-271(3.41)$ \\
vdW-DF2 & $-178(3.80)$ & $-174(3.84)$ \\
DFT/vdW-WF2s1 & $-283(2.98)$ & $-229(3.05)$ \\
DFT/vdW-WF2s1 & {$[-369]$} & {$[-316]$} \\
Expt. $^{\text {a }}$ & {$[-369]$} & - \\
Expt. $^{\mathrm{b}}$ & {$[-317]$} & - \\
Expt. $^{\mathrm{c}}$ & {$[-191 \pm 9]$} & - \\
\hline \hline
\end{tabular}

${ }^{\mathrm{a}}$ Reference [85].

${ }^{\mathrm{b}}$ Reference [86].

${ }^{\mathrm{c}}$ Reference [87].

energy of the interacting system, including the vdW correction term; $E_{s}$ and $E_{\mathrm{Xe}}$ are evaluated using the same supercell adopted for $E_{\text {tot }}$. Note that all the methods predict that the top configuration is favored with respect to the hollow one, in line with the observed general tendency of $\mathrm{Xe}$ and $\mathrm{Kr}$ for adsorption on metallic surfaces in the low-coordination top sites [72,73,75,82,83]. This behavior is attributed [82,84] to the delocalization of charge density that increases the Pauli repulsion effect at the hollow sites relative to the top site and lifts the potential well upwards both in energy and height.

The experimentally measured adsorption energy, $E_{a}$, often includes not only the interaction of adparticles with the substrate but also lateral, vdW interfragment interactions [39,52]. Therefore sometimes it is more appropriate to compare experimental data with the quantity $E_{a}$ which can be related to $E_{b}$ by [52]

$$
E_{a}=E_{b}+\left(E_{l}-E_{f}\right),
$$

where $E_{l}$ is the total energy (per particle) of the twodimensional lattice formed by the adparticles only (that is as in the adsorption configurations but without the substrate and including vdW interfragment corrections when vdW corrected methods are used) and $E_{f}$ is the energy of an isolated (free) adparticle.

Experimental estimates of the adsorption energy for $\mathrm{Xe}$ on $\mathrm{Ni}(111)$ range from -370 to $-180 \mathrm{meV}$ [85-87]. These data were obtained from measurements of the isosteric heat of adsorption [86] and optical differential reflectance studies of adsorption and desorption of $\mathrm{Xe}$ on $\mathrm{Ni}(111)$ [87]. In the latter investigations the overlayer structure at the saturation coverage (one Xe atom per three top $\mathrm{Ni}$ atoms) appears commensurate with the underlying $\mathrm{Ni}(111)$ lattice, which is consistent with the fact that the Xe-Xe separation on $\mathrm{Ni}(111)$ is $4.31 \AA$, which is compressed from the bulk Xe equilibrium value of $4.34 \AA$ by only $0.7 \%$.

Our computed binding energy of Xe on Ni(111) is similar to that obtained by the rVV10 functional, while it is smaller (in absolute value) than the values predicted by LDA and PBE-D and larger than the vdW-DF2 value, and obviously the $\mathrm{PBE}$ one since this functional does not properly include $\mathrm{vdW}$ interactions. Interestingly, by taking the $\mathrm{Xe}-\mathrm{Xe}$ interaction into account to estimate $E_{a}$, our DFT/vdW-WF2s 1 value is compatible with the experimental estimates, while instead LDA and PBE-D appear to significantly overbind. To make the comparison with the experiment more accurate one must point out that, although $\mathrm{Xe}$ on $\mathrm{Ni}(111)$ is considered a typical case of the physisorption process, a net transfer of electronic charge is observed from the Xe atom to the metal, which leads to a surface-induced weakening of the Xe-Xe interaction resulting from the Coulomb repulsion due to the charging of the adatoms [88]. As a result, the absolute value of the correction factor $\left|\left(E_{l}-E_{f}\right)\right|=88 \mathrm{meV}$ in Eq. (12) is probably overestimated; in fact, according to Ref. [87], the difference between $E_{a}$ and $E_{b}$ should be in the range of 30-40 meV. Considering this further correction, our DFT/vdW-WF2s1 estimate for $E_{a}$ would be about $-320 \mathrm{eV}$, always in line with the experimental values.

In Table II we report our computed binding energies and equilibrium distances for graphene on $\mathrm{Ni}(111)$, compared with data obtained by other methods and literature experimental and theoretical estimates; note that in this case much more data are available than for $\mathrm{Xe}$ on $\mathrm{Ni}(111)$. The binding energy curves for graphene on $\mathrm{Ni}(111)$, obtained using our DFT/vdW-WF2s1 method and relative to the three considered configurations, are plotted in Fig. 2.

By focusing on the top-fcc configuration, as discussed above, although not necessarily exact [33], the RPA estimate $[19,20,26]$ can be assumed to be the "best" theoretical estimate to be taken as a meaningful reference. As expected, the PBE functional, which does not include genuine vdW effects, dramatically underestimates the binding so that the binding energy is positive, which means that the system is actually unbound, although the equilibrium distance,

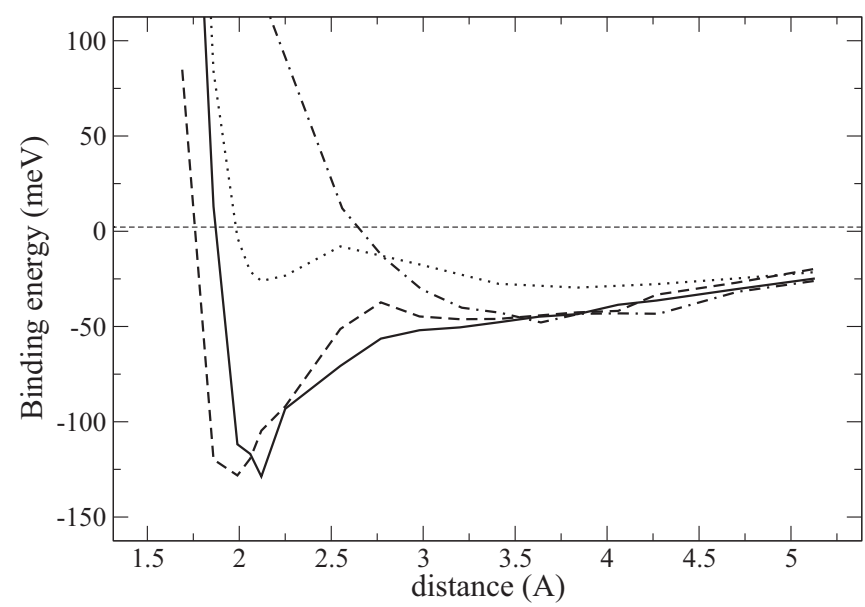

FIG. 2. Binding energy (per $\mathrm{C}$ atom) of graphene on $\mathrm{Ni}(111)$ computed by the DFT/vdW-WF2s1 method, in the top-fcc (solid line), bridge-top (dashed line), and fcc-hcp (dot-dashed line) configurations, as a function of the distance between the graphene layer and the Ni(111) surface. The dotted line has been obtained using the DFT/vdW-WF2s1-shift scheme (see text). 
TABLE II. Binding energy $E_{b}$ (in meV per $\mathrm{C}$ atom; a positive value means that the configuration is unbound) and (in parentheses) equilibrium distance $R$ (in $\AA$ ) for graphene on $\mathrm{Ni}(111)$, considering the top-fcc, bridge-top, and fcc-hcp configurations, using different methods, compared with available experimental and theoretical reference data. $R$ is defined as the separation between the average $z$ coordinate of the $\mathrm{C}$ atoms of the graphene layer and that of the topmost $\mathrm{Ni}$ atoms of the $\mathrm{Ni}(111)$ substrate.

\begin{tabular}{lccc}
\hline \hline Method & top-fcc & bridge-top & fcc-hcp \\
\hline LDA & $-196(2.01)$ & $-210(1.95)$ & $-35(3.24)$ \\
PBE & $+9(2.17)$ & $+9(2.09)$ & $-5(3.85)$ \\
PBEsol & $-131(2.08)$ & $-138(1.99)$ & $-18(3.49)$ \\
DFT-D2 $^{\mathrm{a}}$ & $-160(2.09)$ & $-157(2.05)$ & $-89(3.02)$ \\
DFT-D3 $^{\mathrm{b}}$ & $-107(3.25)$ & - & - \\
rVV10 & $-81(3.14)$ & $-80(3.11)$ & $-75(3.44)$ \\
vdW-DF $^{\mathrm{c}}$ & $-37(3.50)$ & - & - \\
vdW-DF2 $^{\mathrm{d}}$ & $-44(3.68)$ & - & - \\
vdW-DF2 $^{\text {C09 }}{ }_{x}^{\mathrm{d}}$ & $-141(2.07)$ & - & - \\
rev-vdW-DF2 $^{\mathrm{e}}$ & $-75(2.16)$ & - & - \\
M06L $^{\mathrm{f}}$ & $-64(2.29)$ & - & - \\
MGGA_MS2 $^{\mathrm{g}}$ & $-49(2.09)$ & - & - \\
optB86b-vdW $^{\mathrm{h}}$ & $-112(2.12)$ & - & - \\
optB88-vdW $^{\mathrm{i}}$ & $-71(2.18)$ & - & - \\
RPA $^{\mathrm{j}}$ & $-67(2.17)$ & - & - \\
RPA $^{\mathrm{j}}$ & $-70(2.19)$ & - & - \\
DFT/vdW-WF2s1 $_{\text {DFT/vdW-WF2s1-shift }}$ & $-129(2.12)$ & $-128(1.99)$ & $-48(3.64)$ \\
Expt. $^{\mathrm{k}}$ & $-29(3.84)$ & - & - \\
\hline \hline
\end{tabular}

\footnotetext{
${ }^{\mathrm{a}}$ Reference [40].

${ }^{\mathrm{b}}$ Reference [27].

${ }^{c}$ Reference [28].

${ }^{\mathrm{d}}$ Reference [24].

${ }^{\mathrm{e}}$ Reference [25].

${ }^{\mathrm{f}}$ Reference [29].

${ }^{g}$ Reference [23].

${ }^{\mathrm{h}}$ Reference [20].

${ }^{\mathrm{i}}$ Reference [19].

${ }^{\mathrm{j}}$ Reference [26].

${ }^{\mathrm{k}}$ Reference [5].
}

corresponding to a local minimum in the binding energy, agrees well with the reference estimates. However, even the performances of some $\mathrm{vdW}$ corrected functionals are not satisfactory by stressing the difficulty of properly describing this system. In fact, for instance, rVV10 largely overestimates the equilibrium distance and, by comparison with the RPA curve, it appears to completely miss the first (chemisorption) minimum. The vdW-DF and vdW-DF2 methods underestimate the binding energy but (above all) predict a much too large equilibrium distance. Other approaches (see Table II) give reasonable equilibrium distances_-although the estimated binding energies are significantly scattered-with the revvdW-DF2 [25], optB88-vdW [19], and MGGA_MS2 [23] methods, which appear to give the best performances.

For a better assessment of the different approaches, one should remember that RPA tends to underbind [33-35]: for instance, in the case of the interaction of $\mathrm{CO}$ with the $\mathrm{Cu}(111)$ surface, RPA remarkably predicts the correct favored adsorption site; however, it underestimates [35] the adsorption energy by $70 \mathrm{meV}$, corresponding to a significant error of about $15 \%$ with respect to both the experimental reference value and high-level quantum chemistry (CASPT2) calculations. By taking this observation into account and looking at both the binding energy and the equilibrium distance, the performances of DFT/vdW-WF2s1 are rather good, being also better than those of the semiempirical DFT-D2 and DFT-D3 approaches: in fact, DFT-D2 tends to overbind, while DFT-D3 evidently overestimates the equilibrium distance. Interestingly, the DFT/vdW-WF2s1 results are similar to those obtained by the PBEsol functional, but for the fcc-hcp configuration. This behavior is understandable since PBEsol is a semilocal GGA functional [89] (a revision of PBE to better describe solid state and surface systems) which does not properly include vdW interactions and therefore cannot correctly reproduce the energy minimum of the fcc-hcp structure that is essentially determined by long-range vdW effects. Our DFT/vdW-WF2s1 results indicate that the top-fcc configuration is only marginally more favored than the bridge-top, being in line with recent experimental measurements [18,21], which suggest that the total energies of the top-fcc and bridge-top structures are nearly degenerate since the two phases are observed to coexist. Note that LDA and PBEsol, which do not take vdW effects into account, differently from the vdW corrected DFT/vdW-WF2s1, DFT-D2, and rVV10 methods, indicate the bridge-top as energetically more favored than top-fcc, although this result should not be overemphasized, given the relatively small energy differences between the two configurations. Nonetheless it suggests that a proper inclusion of vdW interactions can be of importance even when covalent interactions are involved, as already observed elsewhere [90]. Interestingly, the binding-energy curve of the bridge-top configuration in Fig. 2 exhibits a shallow minimum at about $3.2 \AA$ in addition to the first, more pronounced minimum at shorter distance, in qualitative agreement with the RPA findings $[19,20,26]$ (for the top-fcc configuration this second minimum is not clearly visible).

In Fig. 2, for the top-fcc case only, we also report the binding-energy curve obtained using the DFT/vdW-WF2s1 scheme where, however, at each graphene-Ni(111) distance, the MLWFs used for the calculation of the vdW energy are just those obtained at the largest distance $(5.13 \AA)$ but rigidly shifted along the vertical $z$ coordinate in such a way to be placed at the same $z$ level of the graphene layer (DFT/vdW-WF2s1-shift scheme). As can be seen, this curve is very different from that obtained by considering the MLWFs consistently generated at each graphene-Ni(111) distance; in particular, it predicts that the equilibrium value (see Table II) is at a much larger distance, as expected for a configuration dominated by standard long-range vdW interactions. This clearly demonstrates that the rearrangement of the MLWFs when graphene approaches the $\mathrm{Ni}(111)$ substrate, as a consequence of the bond formation, is indeed crucial for a reasonable description of the energetics of the system. This observation is confirmed by analyzing Fig. 3 where histograms are plotted showing the distribution of the WFCs along the $z$ coordinate [perpendicular to the $\mathrm{Ni}(111)$ surface], by also taking the relative spreads of the MLWFs into account as a Gaussian smearing. By considering, for the top-fcc configuration, two different graphene-Ni(111) distances, representative for the 

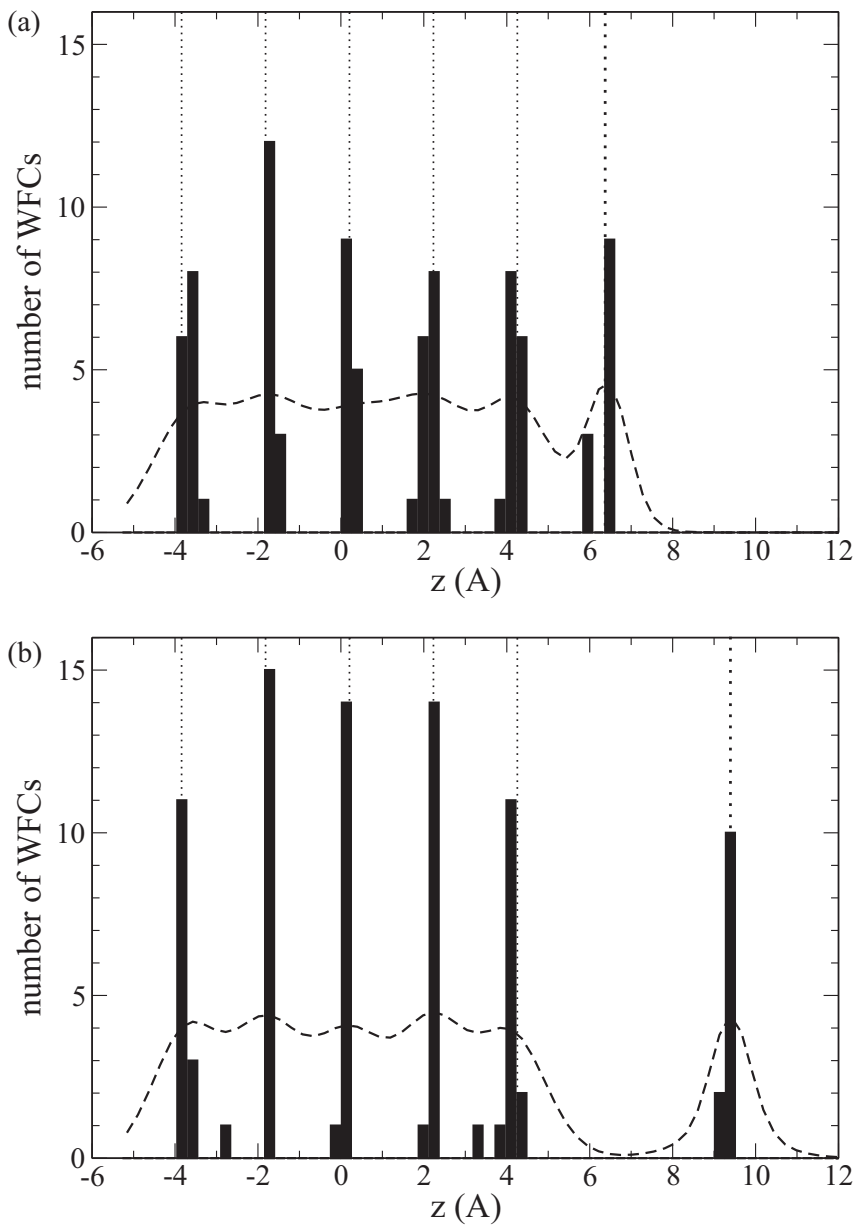

FIG. 3. Histograms showing the distribution of the WFCs along the $z$ coordinate [perpendicular to the $\mathrm{Ni}(111)$ surface] for the top-fcc configuration for graphene on $\mathrm{Ni}(111)$ : (a) corresponds to the binding energy minimum at the $2.12 \AA$ distance, while (b) corresponds to the $5.13 \AA$ distance. The dashed curve has been obtained by a Gaussian smearing where the Gaussian widths are just given by the spreads of the MLWFs, while the vertical dotted lines denote the average position of the $\mathrm{Ni}$ atoms belonging to the different $\mathrm{Ni}(111)$ layers and (thicker line on the right) of the $\mathrm{C}$ atoms of graphene.

chemisorbed structure (corresponding to the binding energy minimum) and for the physisorbed structure (corresponding to the largest distance), respectively, the different distribution is evident. In fact, at large graphene-Ni(111) distance there is a clear separation between the WFCs "belonging" to the graphene layer and those describing the electronic charge distribution of the $\mathrm{Ni}(111)$ substrate, mainly localized at positions along $z$ corresponding to the locations of the metal layers, while at the shorter equilibrium distance, three WFCs, corresponding to MLWFs characterized by larger spreads (about $1.6 \AA$ ) than those of typical MLWFs of freestanding graphene (about $0.8 \AA$ ), are below graphene at an intermediate position between graphene and $\mathrm{Ni}(111)$. By also considering (see Fig. 1) that three is just the number of $\mathrm{C}$ atoms located on top positions and thus able to form covalent bonds with the Ni substrate, within the simulation cell, this feature well illustrates the chemical bonding formation even without the need of a detailed analysis of the electron density distribution, although we must observe that, besides the chemisorption tendency, charge rearrangements neither caused by a charge transfer nor by chemical interactions are also present ("pillow effect") as shown by Gebhardt et al. [31]. Also note that the number of WFCs localized slightly above the topmost Ni layer increases from two to six as the graphene-Ni(111) distance is decreased from 5.13 to $2.12 \AA$, in line with the reported [19] tendency of an electron transfer from the $\mathrm{Ni}(111)$ substrate to adsorbed graphene. Moreover, the dashed curve obtained by the Gaussian smearing clearly suggests that a significant overlap between the MLFWs belonging to graphene and those of the $\mathrm{Ni}(111)$ substrate occurs. Such an overlap is instead absent at the largest distance where evidently no chemical bond between graphene and $\mathrm{Ni}(111)$ is formed.

\section{CONCLUSIONS}

In conclusion, we have extended the applicability of our DFT/vdW-WF2s1 method by investigating the interaction of Xe and graphene with the $\mathrm{Ni}(111)$ transition-metal surface, the latter case representing a challenging scenario for a theoretical description. The results of our calculations, compared with available experimental data and other theoretical investigations, are rather good. We confirm that the rare gas Xe atom is preferentially adsorbed on the top-site configuration on the $\mathrm{Ni}(111)$ surface too, similarly to what happens on other metallic surfaces. Moreover, our approach, based on the use of the MLWFs, allows us to well characterize the bonds between graphene and $\mathrm{Ni}(111)$, which is clearly chemical in character in spite of the relatively small binding energy. This opens the way to applications of the method to investigate adsorption processes also on other interesting transition-metal surfaces such as $\mathrm{Rh}(111)$.

\section{ACKNOWLEDGMENTS}

We thank R. Sabatini for help in performing rVV10 calculations and L. Vattuone for useful discussions. We also acknowledge financial support from the National Research Project (PRIN 2010-2011) GRAF, funded by the Italian Ministry of Education, University, and Research (MIUR).
[1] J. Wintterlin and M. L. Bocquet, Surf. Sci. 603, 1841 (2009).

[2] E. Voloshina and Y. Dedkov, Phys. Chem. Chem. Phys. 14, 13502 (2012).

[3] M. Batzill, Surf. Sci. Rep. 67, 83 (2012).

[4] A. Nagashima, N. Tejima, and C. Oshima, Phys. Rev. B 50, 17487 (1994).
[5] Y. Gamo, A. Nagashima, M. Wakabayashi, M. Terai, and C. Oshima, Surf. Sci. 374, 61 (1997).

[6] M. Fuentes-Cabrera, M. I. Baskes, A. V. Melechko, and M. L. Simpson, Phys. Rev. B 77, 035405 (2008).

[7] W. Zhao, S. M. Kozlov, O. Höfert, K. Gotterbarm, M. P. A. Lorenz, F. Viñes, C. Papp, A. Görling, and H.-P. Steinrück, Phys. Chem. Lett. 2, 759 (2011). 
[8] Y. S. Dedkov, M. Fonin, U. Rudiger, and C. Laubschat, Phys. Rev. Lett. 100, 107602 (2008).

[9] A. Grüneis, K. Kummer, and D. V. Vyalikh, New J. Phys. 11, 073050 (2009).

[10] Yu. S. Dedkov and M. Fonin, New J. Phys. 12, 125004 (2010).

[11] M. Li, J. B. Hannon, R. M. Tromp, J. Sun, J. Li, V. B. Shenoy, and E. Chason, Phys. Rev. B 88, 041402(R) (2013).

[12] A. Allard and L. Wirtz, Nano Lett. 10, 4335 (2010).

[13] P. Riccardi, A. Cupolillo, M. Pisarra, A. Sindona, and L. S. Caputi, Appl. Phys. Lett. 97, 221909 (2010).

[14] Y. S. Dedkov, M. Sicot, and M. Fonin, J. Appl. Phys. 107, 09E121 (2010).

[15] Y. Cho, Y. C. Choi, and K. S. Kim, J. Phys. Chem. C 115, 6019 (2011).

[16] G. Odahara, S. Otani, C. Oshima, M. Suzuki, T. Yasue, and T. Koshikawa, Surf. Sci. 605, 1095 (2011).

[17] A. Garcia-Lekue, T. Balashov, M. Olle, G. Ceballos, A. Arnau, P. Gambardella, D. Sanchez-Portal, and A. Mugarza, Phys. Rev. Lett. 112, 066802 (2014).

[18] F. Bianchini, L. L. Patera, M. Peressi, C. Africh, and G. Comelli, J. Phys. Chem. Lett. 5, 467 (2014).

[19] W. B. Zhang, C. Chen, and P.-Y. Tang, J. Chem. Phys. 141, 044708 (2014).

[20] F. Mittendorfer, A. Garhofer, J. Redinger, J. Klimeš, J. Harl, and G. Kresse, Phys. Rev. B 84, 201401(R) (2011).

[21] D. E. Parreiras, E. A. Soares, G. J. P. Abreu, T. E. P. Bueno, W. P. Fernandes, V. E. de Carvalho, S. S. Carara, H. Chacham, and R. Paniago, Phys. Rev. B 90, 155454 (2014).

[22] X. Sun, S. Entani, Y. Yamauchi, A. Pratt, and M. Kurahashi, J. Appl. Phys. 114, 143713 (2013).

[23] J. Sun, B. Xiao, Y. Fang, R. Haunschild, P. Hao, A. Ruzsinszky, G. I. Csonka, G. E. Scuseria, and J. P. Perdew, Phys. Rev. Lett. 111, 106401 (2013).

[24] I. Hamada and M. Otani, Phys. Rev. B 82, 153412 (2010).

[25] I. Hamada, Phys. Rev. B 89, 121103(R) (2014).

[26] T. Olsen and K. S. Thygesen, Phys. Rev. B 87, 075111 (2013).

[27] X. Li, J. Feng, E. Wang, S. Meng, J. Klimes, and A. Michaelides, Phys. Rev. B 85, 085425 (2012).

[28] M. Vanin, J. J. Mortensen, A. K. Kelkkanen, J. M. Garcia-Lastra, K. S. Thygesen, and K. W. Jacobsen, Phys. Rev. B 81, 081408(R) (2010).

[29] M. Andersen, L. Hornekaer, and B. Hammer, Phys. Rev. B 86, 085405 (2012).

[30] K. Toyoda, K. Nozawa, N. Matsukawa, and S. Yoshii, J. Phys. Chem. C 117, 8156 (2013).

[31] J. Gebhardt, F. Viñes, and A. Görling, Phys. Rev. B 86, 195431 (2012).

[32] V. M. Karpan, P. A. Khomyakov, A. A. Starikov, G. Giovannetti, M. Zwierzycki, M. Talanana, G. Brocks, J. van den Brink, and P. J. Kelly, Phys. Rev. B 78, 195419 (2008).

[33] T. Olsen and K. S. Thygesen, Phys. Rev. Lett. 112, 203001 (2014).

[34] X. Ren, P. Rinke, C. Joas, and M. Scheffler, J. Mater. Sci. 47, 7447 (2012).

[35] J. Harl and G. Kresse, Phys. Rev. Lett. 103, 056401 (2009).

[36] P. Sutter, J. T. Sadowski, and E. Sutter, Phys. Rev. B 80, 245411 (2009).

[37] S. Grimme, J. Comput. Chem. 27, 1787 (2006); V. Barone, M. Casarin, D. Forrer, M. Pavone, M. Sambi, and A. Vittadini, ibid. 30, 934 (2009).
[38] S. M. Kozlov, F. Vies, and A. Görling, J. Phys. Chem. C 116, 7360 (2012).

[39] X. Sun and Y. Yamauchi, J. Appl. Phys. 110, 103701 (2011).

[40] M. Hasegawa, K. Nishidate, T. Hosokai, and N. Yoshimoto, Phys. Rev. B 87, 085439 (2013).

[41] K. E. Riley, M. Pitoňák, P. Jurečka, and P. Hobza, Chem. Rev. 110, 5023 (2010).

[42] A. Tkatchenko, L. Romaner, O. T. Hofmann, E. Zojer, C. Ambrosch-Draxl, and M. Scheffler, MRS Bull. 35, 435 (2010).

[43] J. Klimeš and A. Michaelides, J. Chem. Phys. 137, 120901 (2012).

[44] N. Marzari and D. Vanderbilt, Phys. Rev. B 56, 12847 (1997).

[45] P. L. Silvestrelli, Phys. Rev. Lett 100, 053002 (2008).

[46] P. L. Silvestrelli, J. Phys. Chem. A 113, 5224 (2009).

[47] P. L. Silvestrelli, K. Benyahia, S. Grubisiê, F. Ancilotto, and F. Toigo, J. Chem. Phys. 130, 074702 (2009).

[48] P. L. Silvestrelli, Chem. Phys. Lett. 475, 285 (2009).

[49] P. L. Silvestrelli, F. Toigo, and F. Ancilotto, J. Phys. Chem. C 113, 17124 (2009).

[50] A. Ambrosetti and P. L. Silvestrelli, J. Phys. Chem. C 115, 3695 (2011).

[51] F. Costanzo, P. L. Silvestrelli, and Francesco Ancilotto, J. Chem. Theor. Comput. 8, 1288 (2012); Arch. Metall. Mater. 57, 1075 (2012).

[52] P. L. Silvestrelli, A. Ambrosetti, S. Grubisiĉ, and F. Ancilotto, Phys. Rev. B 85, 165405 (2012).

[53] A. Ambrosetti, F. Ancilotto, and P. L. Silvestrelli, J. Phys. Chem. C 117, 321 (2013).

[54] A. Ambrosetti and P. L. Silvestrelli, Phys. Rev. B 85, 073101 (2012).

[55] P. L. Silvestrelli and A. Ambrosetti, Phys. Rev. B 87, 075401 (2013).

[56] P. L. Silvestrelli, J. Chem. Phys. 139, 054106 (2013).

[57] P. L. Silvestrelli and A. Ambrosetti, J. Chem. Phys. 140, 124107 (2014).

[58] V. G. Ruiz, W. Liu, E. Zojer, M. Scheffler, and A. Tkatchenko, Phys. Rev. Lett. 108, 146103 (2012); W. Liu, A. Tkatchenko, and M. Scheffler, Acc. Chem. Res. 47, 3369 (2014).

[59] M. Dion, H. Rydberg, E. Schröder, D. C. Langreth, and B. I. Lundqvist, Phys. Rev. Lett. 92, 246401 (2004); G. Roman-Perez and J. M. Soler, ibid. 103, 096102 (2009).

[60] T. Thonhauser, V. R. Cooper, S. Li, A. Puzder, P. Hyldgaard, and D. C. Langreth, Phys. Rev. B 76, 125112 (2007).

[61] K. Lee, É. D. Murray, L. Kong, B. I. Lundqvist, and D. C. Langreth, Phys. Rev. B 82, 081101(R) (2010).

[62] R. Sabatini, T. Gorni, and S. de Gironcoli, Phys. Rev. B 87, 041108(R) (2013)

[63] J. P. Perdew, K. Burke, and M. Ernzerhof, Phys. Rev. Lett. 77, 3865 (1996).

[64] R. Eisenhitz and F. London, Z. Phys. 60, 491 (1930).

[65] T. Brink, J. S. Murray, and P. Politzer, J. Chem. Phys. 98, 4305 (1993).

[66] A. Bondi, J. Phys. Chem. 68, 441 (1964).

[67] S. Grimme, J. Antony, T. Schwabe, and C. Mück-Lichtenfeld, Org. Biomol. Chem. 5, 741 (2007); S. Grimme, J. Antony, S. Ehrlich, and H. Krieg, J. Chem. Phys. 132, 154104 (2010).

[68] P. Giannozzi, S. Baroni, N. Bonini, M. Calandra, R. Car, C. Cavazzoni, D. Ceresoli, G. L. Chiarotti, M. Cococcioni, I. Dabo, A. Dal Corso, S. Fabris, G. Fratesi, S. de Gironcoli, 
R. Gebauer, U. Gerstmann, C. Gougoussis, A. Kokalj, M. Lazzeri, L. Martin-Samos et al., J. Phys.: Condens. Matter 21, 395502 (2009).

[69] A. Ferretti, B. Bonferroni, A. Calzolari, and M. Buongiorno Nardelli, WanT code [http://www.wannier-transport.org]; see also A. Calzolari, N. Marzari, I. Souza, and M. Buongiorno Nardelli, Phys. Rev. B 69, 035108 (2004).

[70] Th. Seyller, M. Caragiu, R. D. Diehl, P. Kaukasoina, and M. Lindroos, Chem. Phys. Lett. 291, 567 (1998); M. Caragiu, Th. Seyller, and R. D. Diehl, Phys. Rev. B 66, 195411 (2002).

[71] J. L. F. Da Silva, C. Stampfl, and M. Scheffler, Phys. Rev. Lett. 90, 066104 (2003).

[72] J. L. F. Da Silva, C. Stampfl, and M. Scheffler, Phys. Rev. B 72, 075424 (2005).

[73] J. L. F. Da Silva and C. Stampfl, Phys. Rev. B 77, 045401 (2008).

[74] M. C. Righi and M. Ferrario, J. Phys.: Condens. Matter 19, 305008 (2007).

[75] P. Lazić, Ž. Crljen, R. Brako, and B. Gumhalter, Phys. Rev. B 72, 245407 (2005).

[76] Y. N. Zhang, F. Hanke, V. Bortolani, M. Persson, and R. Q. Wu, Phys. Rev. Lett. 106, 236103 (2011).

[77] E. Abad, Y. J. Dappe, J. I. Martínez, F. Flores, and J. Ortega, J. Chem. Phys. 134, 044701 (2011).

[78] J. L. Fajín, F. Illas, and J. R. B. Gomes, J. Chem. Phys. 130, 224702 (2009).
[79] I. Hamada, Phys. Rev. B 86, 195436 (2012).

[80] F. Perrot and M. Rasolt, J. Phys.: Condens. Matter 6, 1473 (1994).

[81] See, for instance, N. W. Ashcroft, and N. D. Mermin, Solid State Physics (Holt-Saunders International Editions, Philadelphia, 1976).

[82] R. D. Diehl, Th. Seyller, M. Caragiu, G. S. Leatherman, N. Ferralis, K. Pussi, P. Kaukasoina, and M. Lindroos, J. Phys.: Condens. Matter 16, S2839 (2004).

[83] A. E. Betancourt and D. M. Bird, J. Phys.: Condens. Matter 12, 7077 (2000).

[84] P. S. Bagus, V. Staemmler, and C. Wöll, Phys. Rev. Lett. 89, 096104 (2002).

[85] G. Vidali, G. Ihm, H. Y. Kim, and M. W. Cole, Surf. Sci. Rep. 12, 135 (1991).

[86] P. Dolle, M. Alnot, J. J. Ehrhandt, A. Thomy, and A. Cassuto, Surf. Sci. 152-153, 620 (1985); D. Fargues, P. Dolle, M. Alnot, and J. J. Ehrardt, ibid. 214, 187 (1988).

[87] A. Wong and X. D. Zhu, Appl. Phys. A 63, 1 (1996).

[88] J. E. Müller, Phys. Rev. Lett. 65, 3021 (1990).

[89] J. P. Perdew, A. Ruzsinszky, G. I. Csonka, O. A. Vydrov, G. E. Scuseria, L. A. Constantin, X. Zhou, and K. Burke, Phys. Rev. Lett. 100, 136406 (2008).

[90] W. Liu, J. Carrasco, B. Santra, A. Michaelides, M. Scheffler, and A. Tkatchenko, Phys. Rev. B 86, 245405 (2012). 